\title{
Temperate phage-antibiotic synergy across antibiotic classes reveals new mechanism for preventing lysogeny
}

\section{Amany Al-Anany}

McMaster University

Alexander Hynes ( $\square$ hynes@mcmaster.ca )

McMaster University https://orcid.org/0000-0002-7058-6006

\section{Article}

Keywords:

Posted Date: February 23rd, 2022

DOI: https://doi.org/10.21203/rs.3.rs-1386871/v1

License: (c) (1) This work is licensed under a Creative Commons Attribution 4.0 International License.

Read Full License 


\section{Temperate phage-antibiotic synergy across antibiotic classes reveals new mechanism for preventing lysogeny}
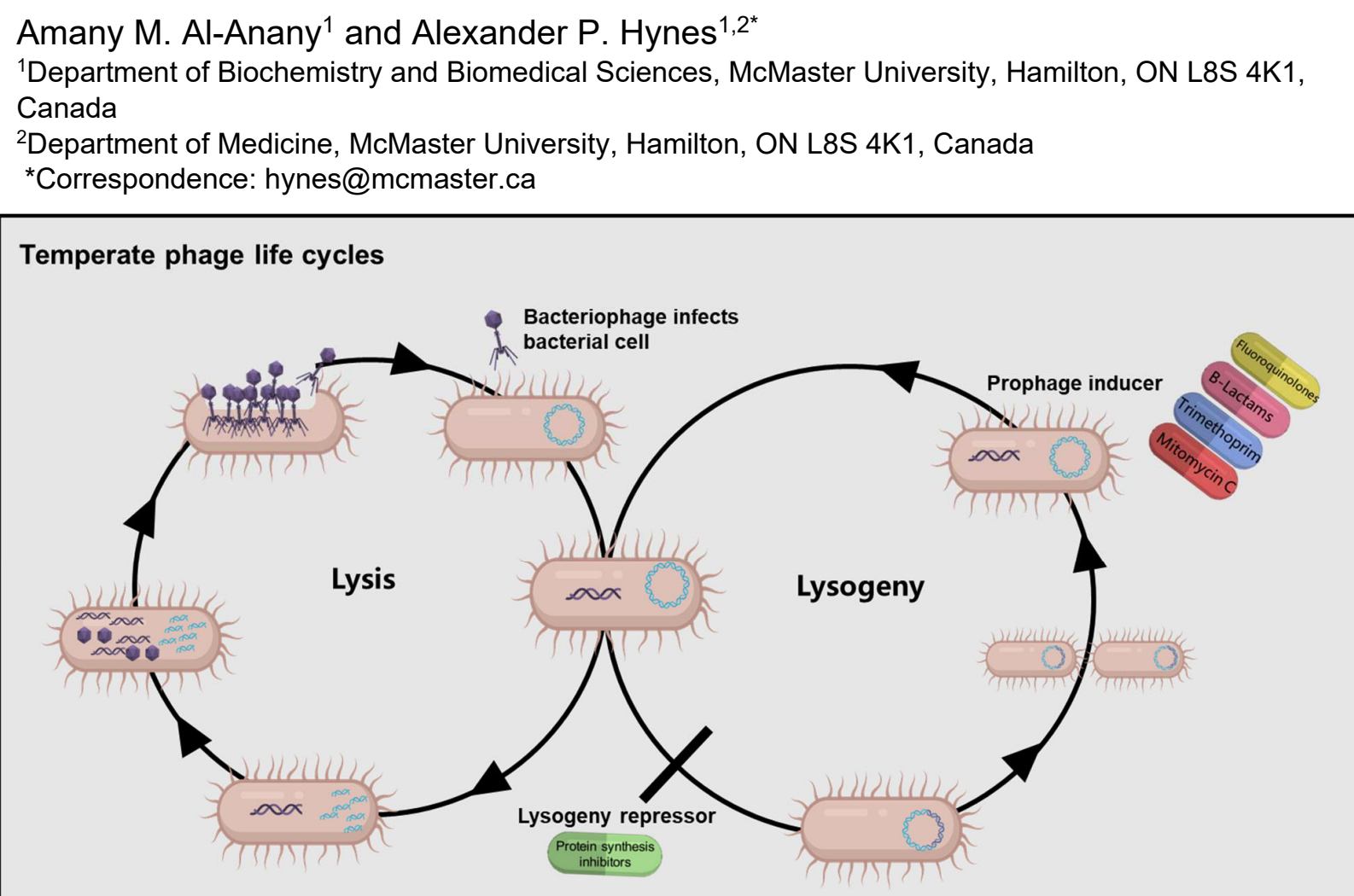

Figure created with Biorender.com.

\section{In Brief}

Al-Anany \& Hynes investigated the synergy of temperate phages with antibiotics across drug classes. Synergy is widely conserved across SOS-inducing agents, as predicted, but also extended to protein synthesis inhibitors, even in the absence of the SOS system. This is the first report of synergy between temperate phages and non-SOS inducing antibiotics, and results from biasing the initial lysis-lysogeny decision. Not only does this have broad implications for the use of temperate phages in therapy, but also reveals an entirely new means of influencing the lysis-lysogeny decision. 


\section{Summary}

A recent demonstration of potent synergy between a temperate phage and the antibiotic ciprofloxacin suggested a scalable approach to exploiting temperate phages in therapy, termed temperate phage-antibiotic synergy (tPAS), which specifically interacted with the lysis-lysogeny decision. To determine whether this would hold true more broadly across antibiotics, we challenged Escherichia coli K12 with the well-studied model $\lambda$-like phage HK97 and a set of 13 antibiotics spanning seven classes. As expected, given the conserved induction pathway targeted, we observed synergy with classes of drugs known to induce an SOS response; a sulfa drug, other quinolones, Mitomycin C, and some $\beta$ lactams. Curiously, we observed an equally potent synergy with antibiotics not known to induce the SOS response: protein synthesis inhibitors gentamicin, kanamycin, tetracycline, and azithromycin. The synergy results in an 8-fold reduction in the effective MIC of gentamicin, complete eradication of the bacteria, and, when deliberately administered at sub-optimal doses, drastically decreases the frequency of lysogens emerging from the combined challenge - more so even than was previously described for ciprofloxacin. However, unlike ciprofloxacin, lysogens exhibit no increased sensitivity to the antibiotic, synergy was maintained in the absence of RecA, and, when quantified over time, the antibiotic reduced the initial frequency of lysogeny rather than result in selection against formed lysogens. Not only do our results confirm that SOS-inducing antibiotics broadly result in temperate-phage specific synergy, but we other antibiotics can interact with temperate phages specifically and result in potent synergy. This is the first report of a means of chemically blocking entry into lysogeny, providing a new means for manipulating the key lysis-lysogeny decision point in temperate phages.

\section{Introduction}

The ongoing crisis of antimicrobial resistance has rekindled interest in bacteriophage (phage) therapy as an alternative to antibiotics, as these bacterial viruses may soon be one of the few remaining options to clear bacterial infections (Cooper et al., 2016). In part out of necessity, because phages must prove themselves alongside the standard of care - antibiotics, and in part to use multiple selective pressures to decrease the emergence of resistance (Gordillo Altamirano and Barr, 2019), phages are often administered alongside antibiotics. These approaches lead to the discovery of phage-antibiotic synergy (PAS).

The term PAS was firstly coined in 2007, when sub-inhibitory concentrations of antibiotic resulted in plaque size enlargement, an increase in phage production and an increase in the latency period (Comeau et al., 2007; Kim et al., 2018). In contrast, Davis et al. (Davis et al., 2021) demonstrated PAS upon utilizing aztreonam lysine in combination with lytic phages E79 and phiKZ characterized with a decrease in infection latency, burst size and accelerated lysis. It was thought at first that PAS effect is primarily dependent on the SOS response induced by these antibiotics (Comeau et al., 2007), but later investigations 
suggested the SOS response plays a minor role and that PAS is a result of alteration in cell morphology in response to the action of these antibiotics (Kim et al., 2018). PAS appears to be broadly applicable, spanning phages across the myoviridae (Chan et al., 2016; Comeau et al., 2007; Jansen et al., 2018; Kebriaei et al., 2020; Kirby, 2012; Liu et al., 2021; Malik et al., 2021; Ryan et al., 2012; Simon et al., 2021; Styles et al., 2020; Wang et al., 2020) siphoviridae (Knezevic et al., 2013; Li et al., 2021), and podoviridae families (Manohar et al., 2022; Save et al., 2022; Styles et al., 2020; Torres-Barceló et al., 2014, 2018; Verma et al., 2009). In addition to phages, PAS has been demonstrated in many hosts: Klebsiella pneumoniae (Bedi et al., 2009; Pacios et al., 2021; Verma et al., 2009), Pseudomonas aeruginosa (Chan et al., 2016; Chaudhry et al., 2017; Coulter et al., 2014; Duplessis et al., 2021; Engeman et al., 2021; Hagens et al., 2006; Oechslin et al., 2017), Actinobacter baumannii (Styles et al., 2020), Staphylococcus aureus (Chhibber et al., 2013; Dickey et al., 2019; Iqbal et al., 2020; Kaur and Chhibber, 2021; Kebriaei et al., 2020; Van Nieuwenhuyse et al., 2021; Yilmaz et al., 2013) and E. coli (Kim et al., 2021; Liu et al., 2020; Moradpour et al., 2020). PAS has also been demonstrated across antibiotics of multiple classes including $\beta$-lactams (Bedi et al., 2009; Comeau et al., 2007; Duplessis et al., 2021; Engeman et al., 2021; lqbal et al., 2020; Moradpour et al., 2020; Ryan et al., 2012), fluoroquinolones (Chaudhry et al., 2017; Comeau et al., 2007; Engeman et al., 2021; Jansen et al., 2018; Knezevic et al., 2013; Oechslin et al., 2017; Torres-Barceló et al., 2014), aminoglycosides (Chaudhry et al., 2017; Coulter et al., 2014; Hagens et al., 2006; Knezevic et al., 2013), and tetracyclines (Iqbal et al., 2020).

In all these studies, PAS was studied in the context of virulent (strictly lytic) phages, as the lysogenic life cycle of temperate phages is considered an insurmountable hurdle for therapy. This is primarily because during lysogeny, the phage integrates its genome into the host and as a result will not have an immediate bactericidal effect (Oppenheim et al., 2005). Second, this cycle affects host fitness and may leave the host with more virulent traits via the integrated prophage, in addition to causing transduction by horizontal gene transfer (Fortier and Sekulovic, 2013). However, this transduction is still common in lytic phages and can not be avoided in phage therapy (Monteiro et al., 2018). Most importantly, lysogeny will typically result in protection from superinfection (Sun et al., 2006). While any antibiotic will select for resistance, a temperate phage will also generate it. This makes lysogeny prevention a key to the eventual success of temperate phages synergy with antibiotics.

Temperate phages usually remain quiescent in the cell unless exposed to a stressor that leads to irreversible switching to a lytic cycle (Howard-Varona et al., 2017). This awakening of dormant phages is known as induction (Lemire et al., 2011) and can happen either spontaneously (Czyz et al., 2001) or as a result of external stressors (HowardVarona et al., 2017). Our understanding of prophage induction primarily stems from wellcharacterized models of lambda and lambdoid (lambda-like) lysogens (Hendrix, 1983; Hendrix et al., 2013). We have recently shown that co-administration of a temperate phage HK97 with a fluoroquinolone antibiotic ciprofloxacin at concentrations below minimum inhibitory concentrations yields potent synergy resulting in bacterial eradication 
( $\geq 9.7 \times 107$ fold reduction) (Al-Anany et al., 2021). This synergy does not greatly increase final phage titers, latency period or burst size, instead greatly reducing the rate of lysogeny - as such, it is distinct from traditional PAS. We coined the term tPAS for synergy with antibiotics that specifically exploits the lysis-lysogeny decision (Al-Anany et al., 2021).

As the interaction between temperate phages and stressors such as ciprofloxacin are known to be widespread across SOS inducing antibiotics (Comeau et al., 2007; Drimmelen, 1959; Lewin and Amyes, 1991; S. Shiba, A. Terawaki, 1952; Simmons et al., 2008), we hypothesized that tPAS could result from the activities of a great many antibiotics. Demonstrating this may enable a safe and scalable approach to allow for the use of these phages in therapy.

\section{Results and discussion}

\section{Generalizability of tPAS across different quinolones}

To test synergy across antibiotics, the standard is a checkerboard assay (Martinez-Irujo et al., 1996), also referred to as synograph (Liu et al., 2020). This previously revealed tPAS reduced the effective ciprofloxacin MIC up to 32-fold, despite the poor effectiveness of phage alone condition (Al-Anany et al., 2021).

To test whether the previously reported synergy was generalizable across other antibiotics, we challenged the same phage E. coli K12-HK97 host-phage pairing used to establish the existence of tPAS (Al-Anany et al., 2021). We first sought to establish whether the reported synergy held true across other antibiotics in the same class as ciprofloxacin, and therefore performed identical checkerboard assays with three other quinolones: nalidixic acid, oxolinic acid and levofloxacin. In these assays, the cut-off value for MIC was calculated for each antibiotic based on antibiotic alone growth curves (S Fig 1 ), with any \%growth value $\leq$ that obtained with the MIC coloured white. Nalidixic acid and phage HK97 yielded a 16-fold reduction in MIC at almost all tested MOls (Figure 1 A). Curiously, there was no clear synergy with either oxolinic acid or levofloxacin (Figure $1 \mathrm{~B}, \mathrm{C})$.

The observation that different antibiotics within the same class result in drastically different synergy profiles concerned us. The SOS-inducing effect of these antibiotics is well-documented (Bellio et al., 2014; N H Georgopapadakou, B A Dix, P Angehrn, A Wick, 1987), as is the association between SOS-induction and phage induction (López et al., 2014; Vondrejs, 1974). We hypothesized that the synergy might be occurring but obscured by endpoint due to the emergence of resistant mutants. Examining the growth of our assays with oxolinic acid at two earlier time points; $9 \mathrm{~h}$ (Figure $1 \mathrm{D}$ ) and $12 \mathrm{~h}$ (Figure $1 \mathrm{E})$ revealed a clear synergistic effect resulting in 8-fold reduction in MIC. Rather than limit ourselves to snapshots in time, we instead opted to monitor continuously (S Fig 2) and represent our data as the area under the curve (AUC) to capture cumulative dynamics of phage antibiotic interaction over time. In these assays, the \%AUC corresponding to the well determined to be MIC (Fig S1) was set as a threshold for a white colour. When the 
heat maps were plotted as a function of \%AUC, we observed a synergistic effect resulting in 32-fold reduction for nalidixic acid (Figure $1 \mathrm{~F}$ ), a 4 -fold reduction in MIC for both oxolinic acid and levofloxacin (Figure $1 \mathrm{G}$ and $\mathrm{H}$ ), and, repeating our prior assays for ciprofloxacin in this manner, found an 8-fold reduction for ciprofloxacin (Figure $1 \mathrm{I}$ ) that was lost in a recA background (Figure $1 \mathrm{~J}$ ).

\section{Endpoint readings after $18 \mathrm{~h}$}

A

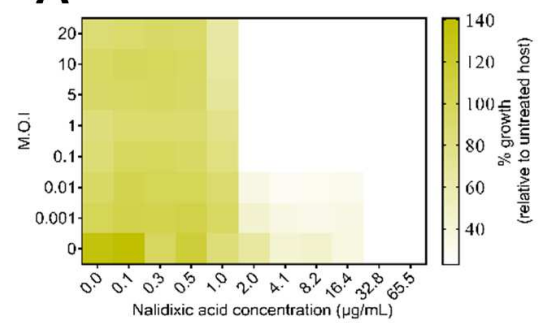

B

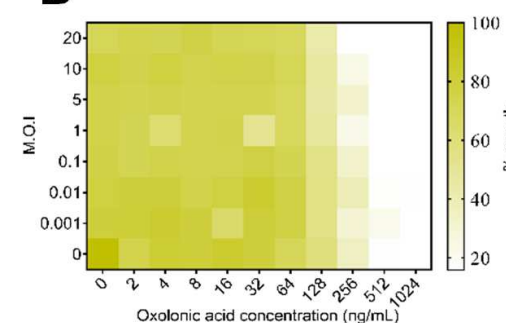

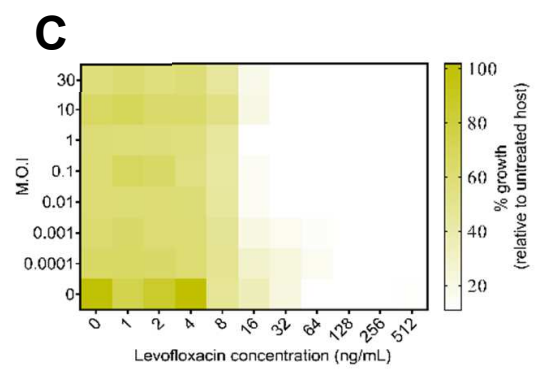

\section{Readings after $9 \mathrm{~h}$ and $12 \mathrm{~h}$}

D

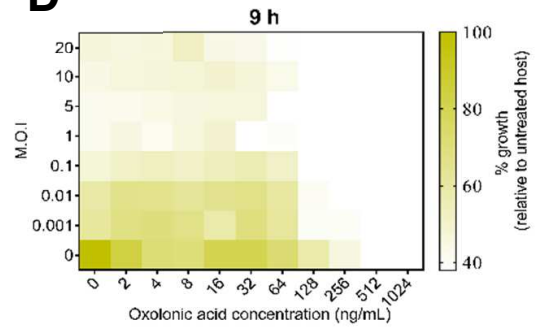

E

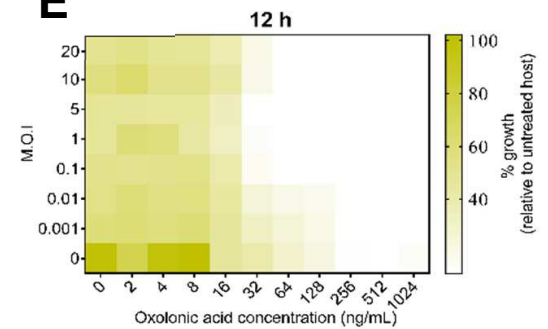

\section{Area under the curve readings}

$\mathbf{F}$

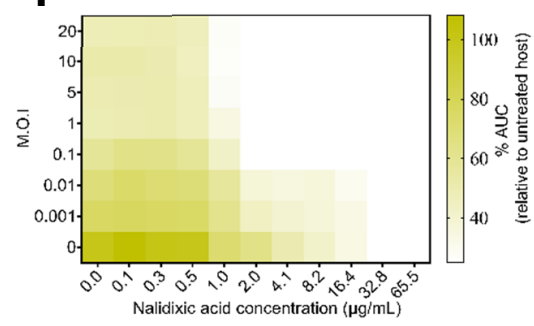

I

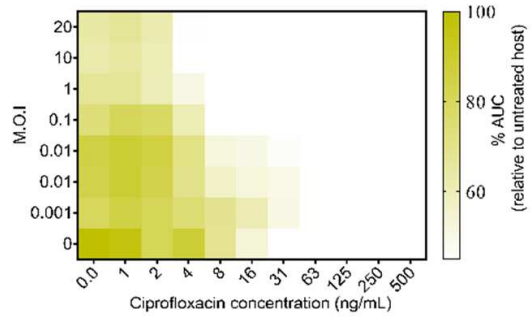

G

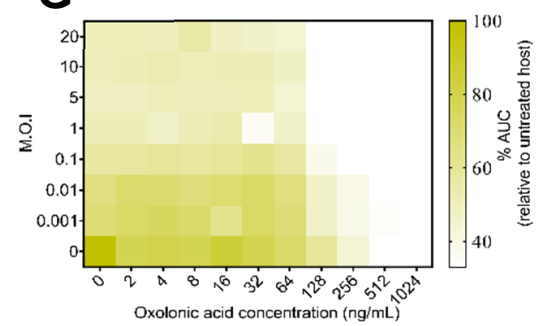

$\mathbf{J}$

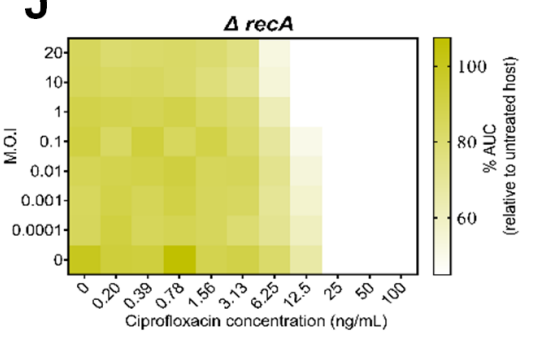

H

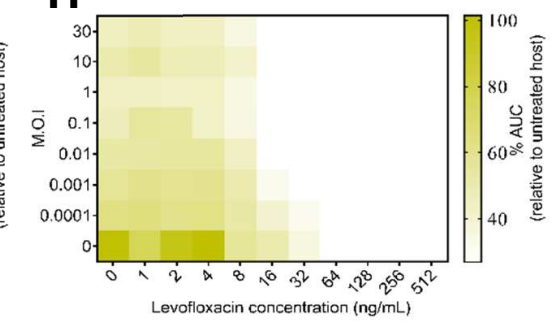

Figure 1. Temperate phage-antibiotic synergy across quinolones. Checkerboard assay of HK97 with quinolones: (A) nalidixic acid, (B) oxolinic acid and (C) levofloxacin. 
replicates, plotted as a heatmap. Readings at $9 \mathrm{~h}$ and $12 \mathrm{~h}$. Checkerboard assay of HK97 and oxolinic acid after $9 \mathrm{~h}$ (D) or $12 \mathrm{~h}$ (E). Growth relative to untreated bacterial control, averaged among 3 biological replicates, plotted as a heatmap. Area under the curve readings. Checkerboard assay of HK97 and (F) nalidixic acid, (G) oxolinic acid, (H) levofloxacin and (I) ciprofloxacin. Area under the curve relative to untreated bacterial control, averaged among 3 biological replicates, plotted as a heatmap. (J) Checkerboard assay of HK97 and ciprofloxacin in recA mutant. Area under the curve relative to untreated bacterial control, averaged among 3 biological replicates, plotted as a heatmap.

Overall, the use of quinolones resulted in synergy, but with different temporal patterns presumably based on the ability of the antibiotic to prevent the long-term regrowth of bacterial survivors.

\section{Test generalizability of tPAS across different antibiotic classes}

Armed with a methodology more sensitive to temporal variations, we moved to investigate tPAS effect across other phage inducing agents, conducting checkerboards with two other antibiotic classes targeting DNA; mitomycin $c$ (DNA damaging agent) and trimethoprim (folic acid synthesis inhibitor). Mitomycin inhibits DNA synthesis and famous as an inducer of temperate phages like lambda and HK97 (Drimmelen, 1959; S. Shiba, A. Terawaki, 1952). Trimethoprim is a sulfa drug that inhibits thymine synthesis by targeting dihydrofolate reductase, which inhibits folic acid synthesis (Amyes and Smith, 1974; Drimmelen, 1959). It has been shown to induce temperate phages in Staphylococcus aureus (Goerke et al., 2006). In Mitomycin C checkerboard (Figure 2 A), we observed synergy like that seen for ciprofloxacin, as expected. This effect resulted in a peak of a 128-fold decrease in mitomycin C MIC at the highest ineffective concentration of phage utilized. While we observed a clear synergistic effect with trimethoprim (Figure 2 B), resulting in an effective 32-fold decrease in trimethoprim MIC, unlike for other antibiotics, synergy was only apparent at phage MOls higher than 1.

$\beta$-lactams are another well-known class of that inhibit cell wall synthesis by binding to a set of membrane proteins known as "penicillin binding proteins" (Bycroft BW, 1985). The presence of $\beta$-lactams is reported to induce the SOS response (Maiques et al., 2006; Miller et al., 2004) and consequently induce phages through the DpiBA two-component signal transduction system (Miller et al., 2004). On one hand, we see no clear synergy with ampicillin or cefotaxime (Figure $2 \mathrm{D}$ and $\mathrm{E}$ ). On the other hand, we observe a clear synergistic effect with cefixime and cefotaxidime (Figure $2 \mathrm{C}$ ) resulting in 4-fold and 16fold reductions in MIC respectively.

Given $\beta$-lactams displayed inconsistent synergy, we hypothesized it might be dependent on the extent to which each antibiotic induces the SOS response. First, to confirm the SOS response was involved, we tested the antibiotics showing clear synergy in our recA mutant. Because RecA plays a role in antibiotic resistance, we first determined the MIC of each drug in our recA mutant (S Fig 3). As with ciprofloxacin (Figure $1 \mathrm{~J}$ ), in the recA background almost all synergy was lost for mitomycin $C$, trimethoprim and for 
cefotaxidime (Figure $2 \mathrm{G}, \mathrm{H}, \mathrm{I}$ ). Where we see tPAS, that synergy is consistently recA dependent.

Figure 2. Temperate phage-antibiotic synergy across antibiotics. Checkerboard assay of HK97 (A) Mitomycin C, (B) Trimethoprim, (C) cefotaxidime, (D) ampicillin, (E)

A

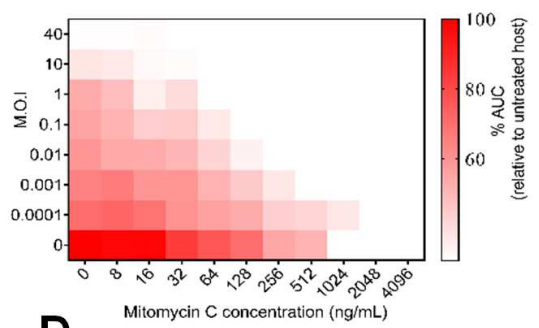

D

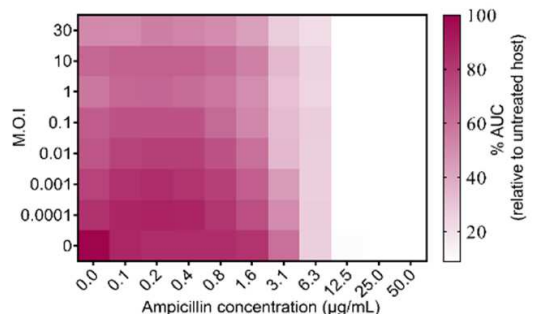

G

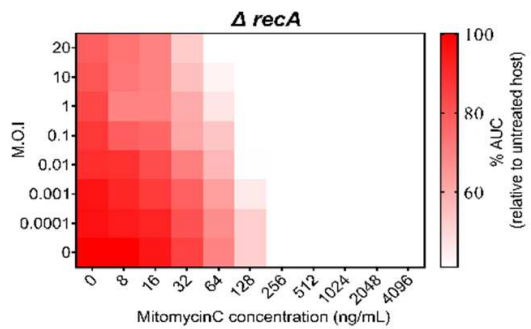

B

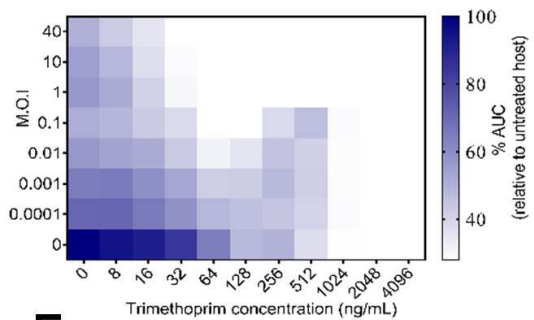

E

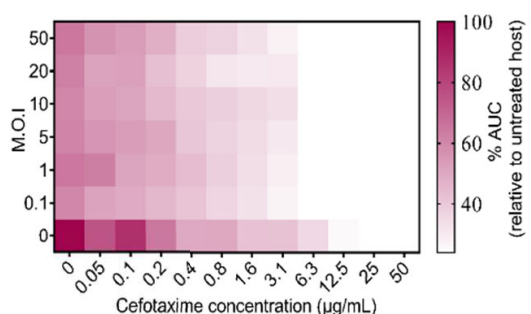

H

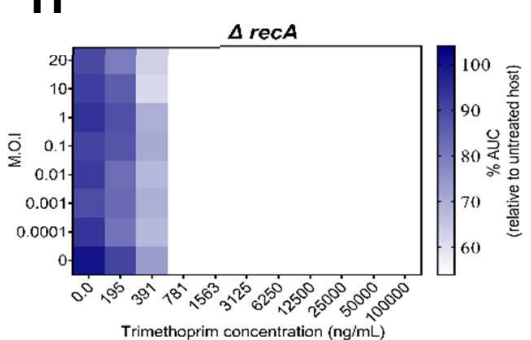

C

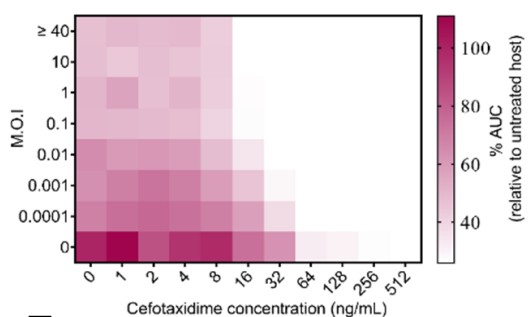

$\mathbf{F}$

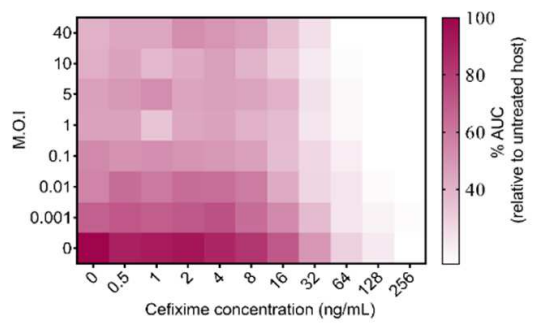

I

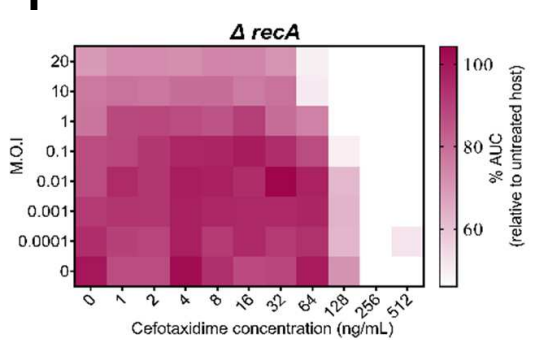

cefotaxime, (F) cefixime. Colour is kept consistent across drugs of the same class. Area under the curve relative to untreated bacterial control, averaged among 3 biological replicates, plotted as a heatmap. Checkerboard assay of HK97 and (G) mitomycin C, (H) Trimethoprim and (I) cefotaxidime in recA mutant. Area under the curve relative to untreated bacterial control, averaged among 3 biological replicates, plotted as a heatmap. 


\section{Assessment of recA activation by antibiotics displaying synergy}

To examine whether the extent of SOS activation plays an important role in our observed synergy, recA and sulA gene expression was studied across antibiotics. The SOS response initiates with the activation of the regulatory protein RecA which will polymerize with ssDNA and consequently induces the autocleavage of LexA (Simmons et al., 2008). This is then followed by a transcription upregulation of the sulA gene which comes further to SOS induction and prevents DNA damage (Simmons et al., 2008). We employed an engineered promoter-reporter gene construct that expresses green fluorescent protein (GFP) upon recA or sulA expression (Southward and Surette, 2002; Zaslaver et al., 2006), normalizing to the fluorescence of pitB, which should not vary based on the SOS response. Gentamicin was added as an antibiotic not known to induce an SOS response. As expected, we observed a significant fold change in recA expression with known SOS inducing antibiotics (Figure $3 \mathrm{~A}$ ) and no change with gentamicin. Interestingly, in ampicillin-treated cultures, we observed the least fold change in $\operatorname{rec} A$ and (Figure $3 \mathrm{~A}$ ) which is not significantly $(p \geq 0.05)$ different from gentamicin nor from no fold change and accordingly we did not reject the null hypothesis. This effect is maintained in sulA expression (Figure 3B). This correlates with a heat map of no synergy (Figure 2D); ampicillin does not seem to induce SOS response enough to trigger a synergy with our temperate phage. In contrast, cefotaxidime, another $\beta$ lactam resulted in 1.7-fold change at $1 / 2$ MIC (Figure $3 \mathrm{~A}$ ) at $1 / 2$ MIC and 1.5 -fold change at all other concentrations which appears to be sufficient for SOS induction to give a synergy with HK97 phage (Figure 2C). These results suggest that there is a minimum threshold for SOS activation needed to induce phages and result in a synergistic effect, but that threshold is best measured by the early SOS response $(\operatorname{rec} A)$ - and that Ampicillin does not cross that threshold.

A

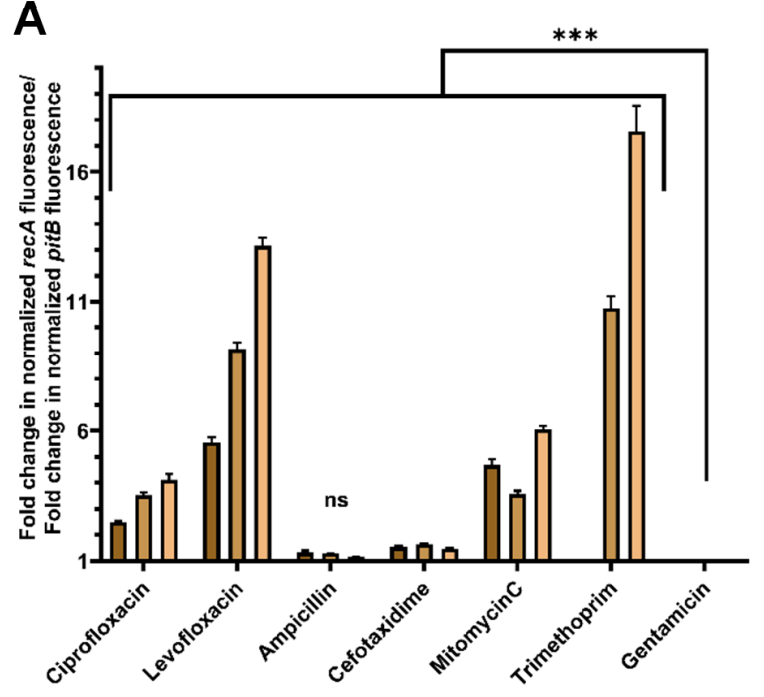

B

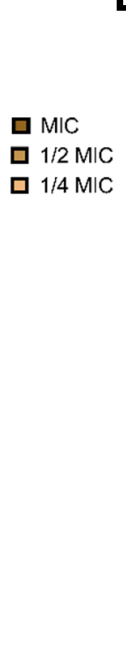

B

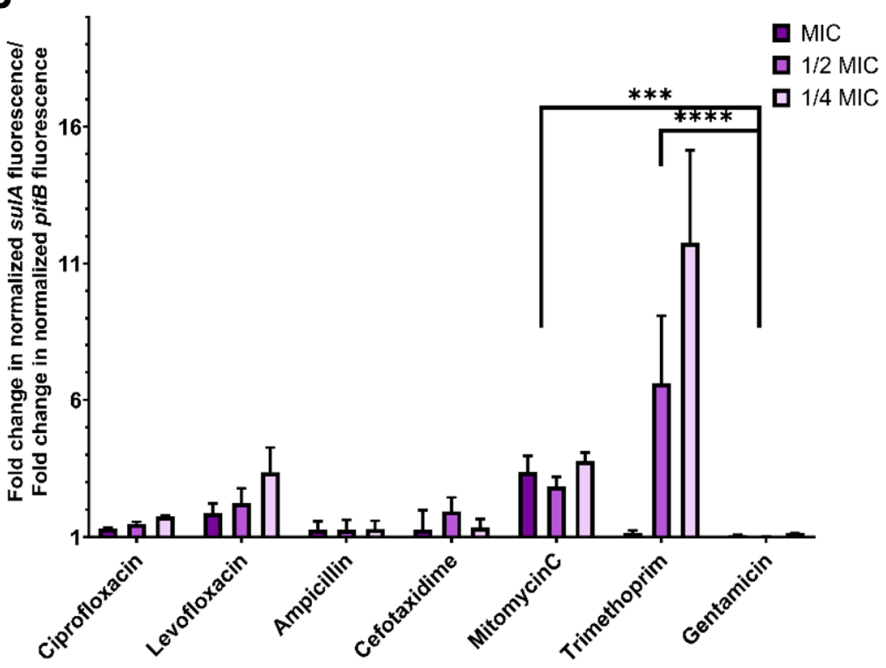

224 Figure 3. $\operatorname{rec} A$ and sulA reporter fluorescence fold change (A) Bars show average normalized fold change in $r e c A$ reporter fluorescence relative to average normalized fold change in pitB reporter fluorescence in 3 technical replicates. (B) Bars show average 
normalized fold change in sulA reporter fluorescence relative to average normalized fold change in pitB reporter fluorescence in 3 technical replicates. Note that the $\mathbf{y}$ axis starts at 1; in both graphs, over 1-fold change would indicate an increase in normalized fluorescence compared to control pitB. Fold change in three technical triplicates with error bars depict the SD was compared using 2-way ANOVA and Tukey multiple comparison tests with $p \geq 0.05$ is no significance, $p^{* * *}$ from 0.0001 to 0.001 and $p^{* * *} \leq 0.0001$.

\section{tPAS in non-SOS inducing antibiotics}

Since cefotaxidime had low SOS activation yet a reasonable synergy, we wanted to ensure gentamicin - which did not detectably induce the SOS response in our assays (Figure 3A and B) - would not result in synergy. Aminoglycosides like gentamicin are not known to induce phages in E. coli (Baharoglu and Mazel, 2011), and, by inhibiting protein synthesis upon which the phage depends, might even antagonize the phage (Drulis-Kawa et al., 2013). In fact, a recent preprint demonstrated that kanamycin impaired phage infection by temperate phage lambda (Luthe et al., 2021).

To confirm this effect with HK97 and demonstrate that non-inducing agents will not result in a synergy, we repeated our checkerboards with two aminoglycosides, a tetracycline, and a macrolide. Unexpectedly, the use of HK97 with either gentamicin, kanamycin, tetracycline, or azithromycin yielded a clear synergistic effect that resulted in efficient inhibition of bacterial growth (Figure 4A, B, C and D). HK97 at MOI $\geq 0.1$ resulted in an 8-fold reduction in MIC of gentamicin, 16-fold with kanamycin or tetracycline and 8-fold reduction in $\mathrm{MIC}$ of azithromycin, although in the latter only at $\mathrm{MOI} \geq 1$. The use of protein synthesis inhibitors with temperate phage HK97 results in a synergy through an unknown mechanism.

As this directly contradicts the findings of Luthe et al with phage lambda, we repeated some of our protein synthesis inhibitors checkerboards in both lambda its virulent mutant lambdavir. Unlike in their work neither kanamycin nor gentamicin yielded antagonism with either phage (Fig S4), although we also did not observe synergy. Their work was done in aminoglycoside resistant strains, with the antibiotic-modifying enzyme AphA1 responsible for phosphorylation of the antibiotic. This could be separating a phage synergizing effect (the antibiotic effect) from a lambda-specific inhibition by kanamycin.

To quantify our synergy between protein synthesis inhibitors and HK97, we opted to focus on gentamicin as it had the strongest synergistic effect with very low phage concentrations, $\mathrm{MOI}$ of 0.01 , resulting in 4-fold reduction in MIC. Survivors arising from unchallenged host, phage challenge, gentamicin challenge, and phage + gentamicin challenge were counted after overnight incubation in liquid media. This assay revealed bacterial eradication at MIC and $1 / 2$ MIC gentamicin. In a dose dependent synergy, the killing effect decreased with decreasing the antibiotic concentrations (Figure 4E); with a more than $4 \log$ reduction in the number of survivors at $1 / 4 \mathrm{MIC}$ compared to untreated 
host compared to 1 - fold reduction with phage challenge alone. This synergy is up to 85fold greater than the multiplicative effects of the phage and antibiotic alone (Figure 4F).

As with the other antibiotics, we tested our synergy in our recA mutant. As expected, neither gentamicin nor tetracycline synergy was wholly dependent on the RecA (Figure 4G and H). This is consistent with the literature (Kohanski MA et al., 2007) and with our findings that gentamicin didn't detectably induce recA or sulA (Figure $3 \mathrm{~A}$ and $\mathrm{B}$ ).

A

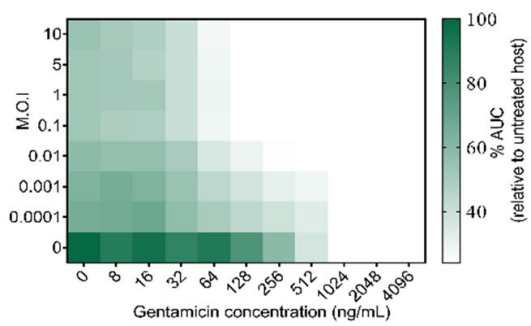

D

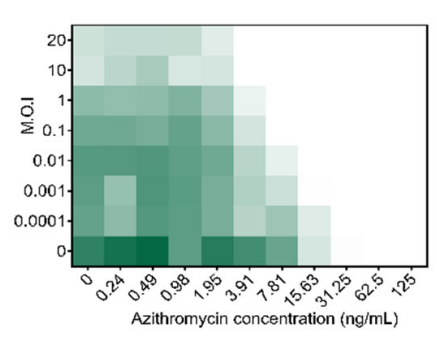

G

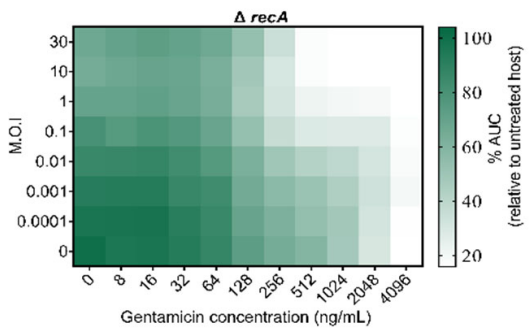

B

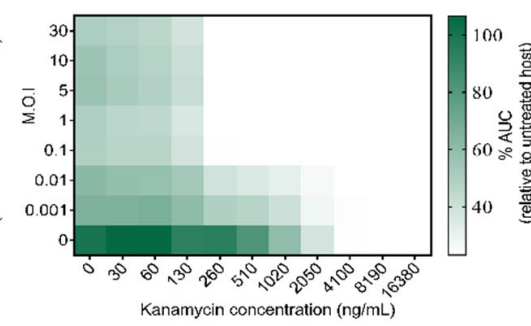

E

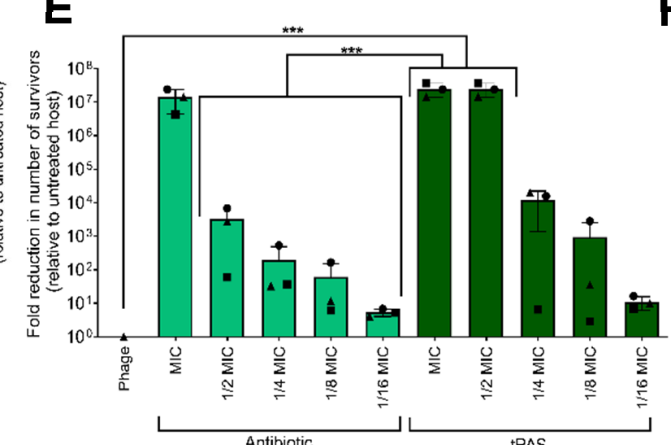

H

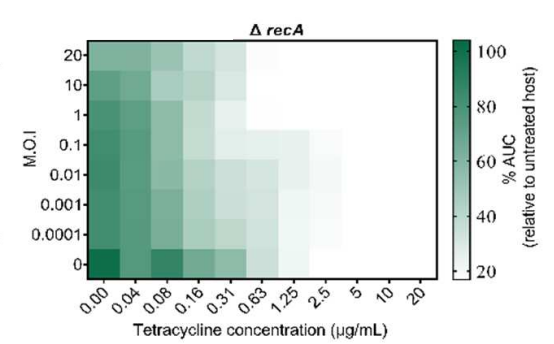

C

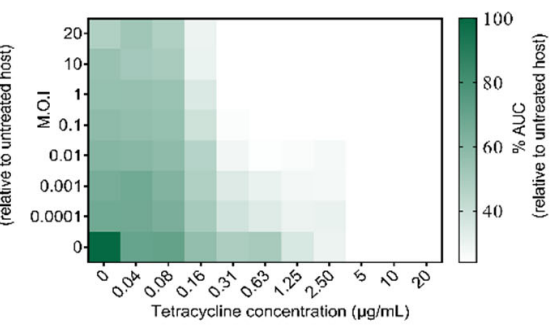

F

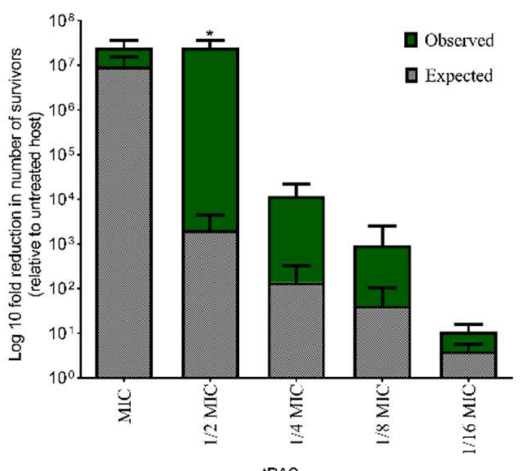

Figure 4. Protein synthesis inhibitors result in temperate phage synergy Checkerboard assay of HK97 and: (A) gentamicin, (B) kanamycin, (C) tetracycline and (D) azithromycin. Area under the curve relative to untreated bacterial control, averaged among 3 biological replicates, plotted as a heatmap. (E) Bars show average number of survivors relative to untreated cultures in 3 biological replicates, each of 3 technical replicates. Each biological replicate is represented by its own shape: circle, square, or triangle. LOD (10 CFU/mL) is represented at all points of MIC and $1 / 2$ MIC tPAS data, as no counts were obtained, except "square" at $1 / 2$ MIC. Error bars depict the SD, while *** p from 0.001 to 0.0001 , from a 1- way ANOVA and Tukey post hoc test. (F) Bars show observed effect (green) versus expected (gray) effect determined by multiplying the effect of the phage and antibiotic alone. Average from the 3 biological replicates for each 
observed tPAS data from Figure 4E was compared to the calculated expected effect at the corresponding antibiotic concentration using paired $t$ test, ${ }^{*} p \leq 0.05$. Checkerboard assay of HK97 and: $(\mathbf{G})$ gentamicin and $(\mathbf{H})$ tetracycline in a recA mutant. Area under the curve relative to untreated bacterial control, averaged among 3 biological replicates, plotted as a heatmap.

\section{Mechanism of tPAS in protein synthesis inhibitors}

Knowing this synergy is largely RecA-independent, we had to confirm that the synergy obtained with protein synthesis inhibitors is tPAS and not PAS independent of the lysislysogeny decision. We first established whether the synergy was acting to reduce the frequency of lysogeny in survivors. Through PCR of the phage-host junction to confirm integration of HK97, we screened purified survivors arising from the challenge at $1 / 4 \mathrm{MIC}$, where we started seeing our first survivors, as well as at $1 / 8 \mathrm{MIC}$. The antibiotic at $1 / 4 \mathrm{MIC}$ reduced the percent of lysogeny from $92 \%$ ( $n=55$ survivors) in the phage-alone challenge to $2 \%(n=55)$ and reducing the antibiotic to $1 / 8$ MIC restored lysogeny rates to $84 \%$ $(n=55)$ (Figure 5A). These results show an efficiency in lysogeny reduction that exceeds that in our previous work with ciprofloxacin (Al-Anany et al., 2021).

Interestingly, the sole lysogen obtained at $1 / 4 \mathrm{MIC}$ was resistant to $\mathrm{HK} 97$ infection through impeded adsorption (Figure 5B). While surface receptor mutation is the most common resistance mechanism in vitro (Labrie et al., 2010), and present in all tested non-lysogens (Fig 5B), this is unusual in a lysogen because it should be protected from subsequent infections through superinfection immunity conferred by the HK97 $\mathrm{cl}$ repressor. We hypothesized that gentamicin might be reducing the effectiveness of superinfection immunity, but superinfections of lysogens in the presence of sub-inhibitory gentamicin yielded no plaques (not shown).

Next, we first sought to establish whether gentamicin was inducing lysogens through some unknown pathway, comparing the antibiotic sensitivity of the lysogen to the parent bacterium. MIC determination curves were found to be similar between the lysogen and the parent bacterium (Figure 5C), and no change in growth was observed upon exposure of the lysogen and non-lysogen to $1 / 2$ MIC gentamicin (Figure 5D). This was accompanied by no change in HK97 phage titer at $2 \mathrm{~h}$ or $6 \mathrm{~h}$ post-exposure at both MIC and $1 / 2 \mathrm{MIC}$ (Figure 5E).

Gentamicin is clearly decreasing the rate of lysogeny (Figure 5A) but has no effect on the number of phages produced by a lysogen (Figure 5E). Moreover, the lysogen has no increased sensitivity to gentamicin (Figure $5 \mathrm{C}$ and D), a characteristic property of phageinducing antibiotics. If gentamicin is reducing the frequency of lysogens but, unlike with ciprofloxacin, not doing so by selecting against them, it must be instead biasing the initial lysis-lysogeny decision. To investigate this hypothesis, culture was challenged with HK97 alone or HK97 with gentamicin at $1 / 2$ MIC, incubated overnight. Samples were then treated with DNase to remove any extracellular free-floating DNA, whether phage or bacterial from already lysed cells. We then extracted genomic DNA and followed the frequency of lysogeny over time by qPCR primers for the HK97-host junction. As early as the $2 \mathrm{~h}$ mark, 
the ratio of lysogens to non-lysogens was significantly lower in the presence of the antibiotic, and this trend persisted out to endpoint at $18 \mathrm{~h}$ (Figure 5F). This is in direct contrast to ciprofloxacin, whose detectable effect on the ratio of lysogens to non-lysogens was only seen after $6 \mathrm{~h}$ (Al-Anany et al., 2021), and supports our claim that gentamicin is biasing the initial lysis-lysogeny decision, in an entirely SOS-dependent manner.

This is not the first report of factors that can force a lytic cycle outside the SOS-response; overexpression of capsular polysaccharide synthesis genes $r c s A$ and $d s r A$ cause lambda and lambdoid prophages induction in a recA mutant (Rozanov et al., 1998), and induction can also controlled be autoinducers (Ghosh et al., 2009), internal ionic environment (Shkilnyj and Koudelka, 2007), EDTA exposure (Imamovic and Muniesa) and micropollutants (Danovaro and Corinaldesi, 2003). Moreover, oral administration of commonly prescribed drugs and dietary products can induce phages, although the SOSresponse was not ruled out (Boling et al., 2020; Sutcliffe et al., 2021; Yasukawa et al., 2002). However, all these studies revealed induction, rather than a specific prevention of lysogeny. Interestingly, we have uncovered an entirely new SOS-independent way of manipulating phage behavior and biasing the initial lysis-lysogeny decision. Fortuitously, this results in potent tPAS. As this synergy appears to exist across aminoglycosides tested, as well as in tetracycline and azythromicin - other protein synthesis inhibitors we suspect that it arises from a delay in the accumulation of lysogeny-favoring proteins - 
likely CII (Herskowitz and Hagen, 1980), therefore greatly decreasing the likelihood of lysogeny.

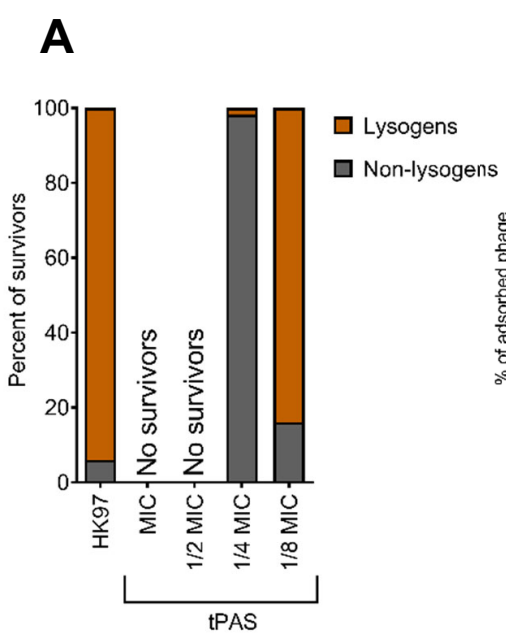

D

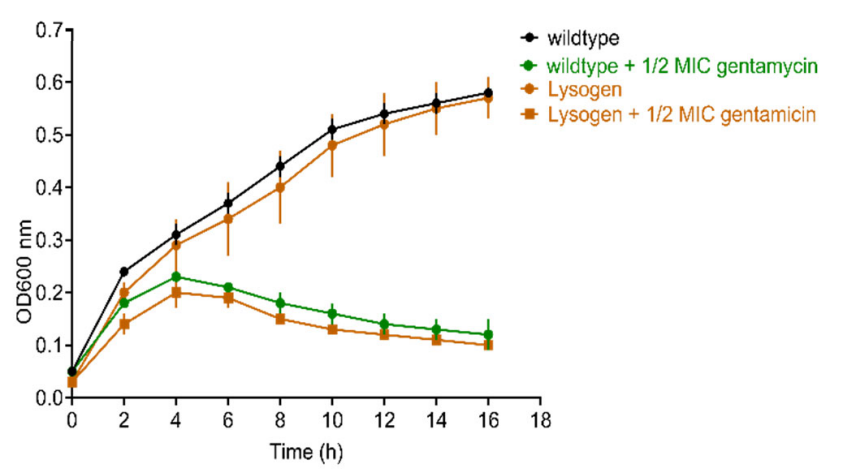

$\mathbf{F}$

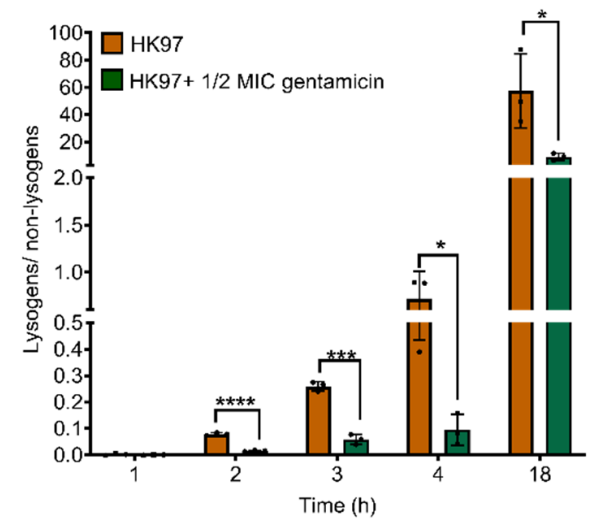

C

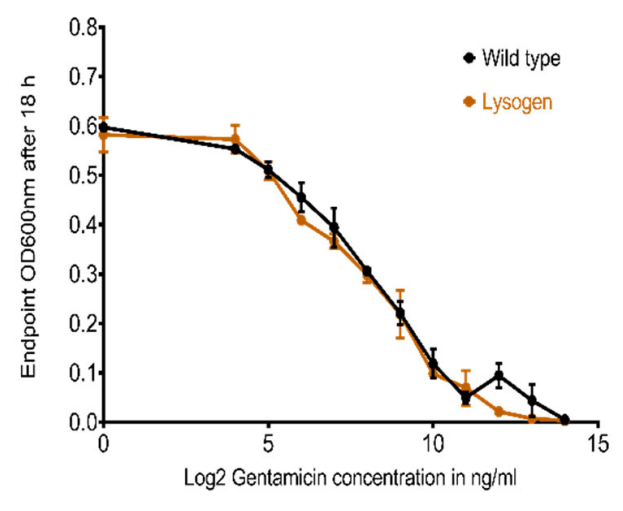

E

Figure 5. Mechanism of gentamicin HK97 synergy. (A) Percentage of lysogen and non-lysogen survivors after overnight HK97 and gentamicin challenges averaged. PCR was performed in duplicate for confirmation. (B) Phage adsorption of survivors. Bars showing $\%$ of adsorbed phage in 4 survivors from $1 / 4$ MIC gentamicin phage challenge; grey are non-lysogens and orange is the single lysogen survivor. Error bars represent SD. Percent was compared using 2-way ANOVA and Tukey multiple comparison tests * $P$ 
50.05. (C) MIC in liquid culture for wild-type E. coli K-12 and lysogen control was tracked after challenging with serial dilutions of ciprofloxacin (means $\pm S D, n=3$ ). MIC is $\sim 1.024 \mu \mathrm{g} / \mathrm{mL}$ for wild type and lysogen. (D) Growth curves in liquid culture of wild-type E. coli K-12 and lysogen control tracked in the absence and presence of gentamicin at $1 / 2$ MIC, averaged among 3 biological replicates \pm SD. (E) Phage quantification at time 0 for lysogen control and after $2 \mathrm{~h}$ and $6 \mathrm{~h}$ when challenged with serial dilutions of gentamicin, averaged among 3 biological replicates, each of 3 technical replicates. Error bars represent SD. Each biological replicate is represented by its own shape: circle, square, or triangle. Titer was compared using 2-way ANOVA and Tukey multiple comparison tests with $p \geq 0.05$ is no significance. (F) Ratio of lysogens to non-lysogens tracked over time for the HK97 or the HK97 and gentamicin challenge using qPCR at 5 time points $(n=3$ performed in biological triplicates) for $1 / 2$ MIC antibiotic. Error bars represent SD. Lysogen/non-lysogen ratio compared using multiple unpaired t tests ${ }^{*} \mathrm{P} \leq 0.05$, ${ }^{* * *} \mathrm{p}$ from 0.001 to 0.0001 and ${ }^{* * * *} p \leq 0.0001$. 
This paper is the first demonstration of the broad applicability of temperate phage synergy with not only SOS-inducing but also non-SOS inducing antibiotics. We demonstrate that temperate phage HK97 can lower the effective MIC of antibiotics belonging to seven different drug classes. This could scalably enable use of temperate phages in therapy, either as adjuvants to antibiotics, or in combination with non-antibiotic inducers (e.g. Boling et. al. 2020). Excitingly, we uncovered the first way to completely block entry to lysogeny: protein synthesis inhibitors. These do not act as phage inducers, and by enabling us to separate the entry into lysogeny from its exit, provide us with a powerful tool to study the lysis-lysogeny decision - arguably the single most important decision point in microbiology - in depth. 


\section{Experimental model and subject details}

The temperate phages model used for this study is lambdoid phage HK97 and lambda. Lambda(vir) was used as a lytic phage model. Escherichia coli K-12 (Ymel mel-1 supF58) and the E. coli BW25113 recA mutant, obtained from the Dharmacon KEIO collection through Horizon Discovery (Cambridge, UK), are the two hosts used for our phages. The Escherichia coli K-12 host, and phages were obtained from Félix d'Hérelle Reference Center for Bacterial Viruses under the accession HER 1382 and HER 382, respectively, with $\lambda$-vir (HER37) propagated on the same host. Bacterial culture was grown as previously described (Al-Anany et al., 2021). Briefly, growth was in $10 \mathrm{~mL}$ lysogeny broth (LB) at $37^{\circ} \mathrm{C}$ with shaking at $130 \mathrm{rpm}$ (Ecotron, Infors HT, Quebec, Canada). For same day use, overnight cultures were diluted 1:100 in LB broth and grown to OD600 0.2 , measured using the Thermo Fischer Scientific Spectronic 20D+ (Waltham, MA, USA).

\section{Method details}

\section{Phage propagation and titration}

Phage lysates were obtained by primary amplification through inoculating frozen bacterial and phage stocks in $10 \mathrm{~mL}$ LB broth growing for a maximum of $18 \mathrm{~h}$, or secondary amplification by inoculating $50 \mu \mathrm{L}$ of previously prepared phage lysate into $10 \mathrm{~mL}$ of grown culture followed by incubation at $37^{\circ} \mathrm{C}$ for up to $4 \mathrm{~h}$. Cultures were then passed through a $0.45 \mu \mathrm{m}$ Basix ${ }^{\mathrm{TM}}$ Syringe Filters, PES, Sterile from Thermo Fisher Scientific filter to obtain a phage lysate. Phage titration was carried out using the double agar overlay technique (Kropinski et al., 2009). Three hundred microliters of overnight grown bacterial culture and $100 \mu \mathrm{L}$ of ten-fold serial dilutions of the lysate prepared in LB were mixed into molten soft agar $0.75 \%(\mathrm{wt} / \mathrm{vol})$ and distributed onto solid $1 \%(\mathrm{wt} / \mathrm{vol})$ agar. Plaques were counted as zones of clearing in bacterial lawn after overnight incubation. Multiplicity of infection (MOI) was determined using the formula: phage titre $(p$ fumL $) \times$ phage volume / colony forming unit $($ cfumL $) \times$ bacterial volume $(m L)$

\section{MIC determination}

MIC of antibiotics was determined using a slightly modified broth dilution method (Wiegand et al., 2008). Briefly, $100 \mu \mathrm{L}$ of freshly grown culture, a volume of antibiotic stock solution, and nuclease free water were combined in a microtiter plate to obtain a final volume of $250 \mu \mathrm{L}$. The microtiter plate was incubated for $18 \mathrm{~h}$ at $37^{\circ} \mathrm{C}$ overnight with double orbital shaking at a frequency of $205 \mathrm{cpm}(5 \mathrm{~mm})$ using Epoch 2 microplate spectrophotometer (BioTek Instruments, Inc., VT, USA). MIC determination was performed in triplicate and end point $\mathrm{OD}_{600 \mathrm{~nm}}$ was measured after $18 \mathrm{~h}$. The MIC would be the lowest concentration of antibiotic in which the final OD was equal to the initial read at time zero. 


\section{Checkerboard assay}

One Hundred microliters of cultures grown till an $\mathrm{OD}_{600}$ of 0.2 were transferred into wells in a 96-well plate containing $100 \mu \mathrm{L}$ of previously diluted phage lysate in LB to achieve target MOls on the vertical axis. In all our checkerboards we started horizontally with the highest antibiotic concentration of at least MIC and then we performed a 2-fold serial dilution till we reach a well of no treatment control with a final volume of $250 \mu \mathrm{L}$. Synergy testing was performed in triplicate, in which the optical density was monitored every 15 mins for $18 \mathrm{~h}$ using an Epoch 2 microplate spectrophotometer, followed by \% growth measurements calculated as follow: [ODtreatment - ODgrowthcontrol / ODgrowth control ] * 100. Results were graphically represented in a heatmap. Our threshold value for heatmaps was calculated for each antibiotic based on antibiotic alone conditions growth curves (See Fig S1). Cut-off value was selected based on \% growth value relative to untreated host at MIC for endpoint checkerboards and \%AUC relative to untreated host value for timepoint measurements heatmaps.

\section{Fluorescence assay}

Two E. coli strains with engineered promoter-reporter gene construct that expresses green fluorescent protein (GFP) upon recA or pitB expression was obtained from the Brown Lab at McMaster university which is originally obtained from (Zaslaver et al., 2006). Hundred microliters of freshly grown cultures untreated or treated with MIC, $1 / 2 \mathrm{MIC}$ and $1 / 4$ MIC concentration of antibiotic (ciprofloxacin, levofloxacin, mitomycin C, cefotaxidime, trimethoprim and gentamicin) were added to a 96-microplates for fluorescence-based assays. Twenty-five microliters of volume of kanamycin $500 \mu \mathrm{g} / \mathrm{ml}$ stock solution were added for strain selection and nuclease free water were combined in a microtiter plate to obtain a final volume of $250 \mu \mathrm{L}$. The microtiter plate was incubated for $18 \mathrm{~h}$ at $37^{\circ} \mathrm{C}$ overnight with double orbital shaking at a frequency of $205 \mathrm{cpm}(5 \mathrm{~mm})$ using Agilent BioTek Synergy Neo2 multimode microplate reader (BioTek synergy Neo2 Instruments, Inc., VT, USA). Resulted fluorescence was initially normalized to OD growth, then fold change compared to untreated host fluorescence was calculated. Normalized fold change in recA fluorescence was then plotted relative to average normalized fold change in pit $B$ in 3 technical replicates.

\section{Broth growth curve}

Growth curves in liquid culture for challenged and non-challenged wild-type E. coli K-12 and E. coli K-12 HK97 lysogen were recorded as follows. Freshly grown cultures were treated with $1 / 2$ MIC concentration of gentamicin $(512 \mathrm{ng} / \mathrm{mL})$. Cultures were then incubated overnight with double orbital shaking and readings were taken every 15 min with the Epoch 2 microplate spectrophotometer.

\section{Overnight quantification assay}


In a $1.5 \mathrm{~mL}$ microcentrifuge tube, $100 \mu \mathrm{L}$ of freshly grown cultures, $100 \mu \mathrm{L}$ of phage lysate for a final $\mathrm{MOI}$ at least 10 and two-fold serial dilutions of antibiotics were added to a final volume of $350 \mu \mathrm{L}$ and mixed by pipetting. Cultures were then incubated overnight with shaking at $130 \mathrm{rpm}$ (Ecotron, Infors HT, Quebec, Canada). Subsequently, a 10-fold serial dilution of each trial was prepared, inoculated in $5 \mathrm{~mL}$ of LB soft agar $(0.75 \%)$ and then incubated overnight. Survivors from each challenge were counted and the actual number of survivors in $1 \mathrm{~mL}$ broth was calculated. Subsequently, fold reduction compared to the untreated host was calculated as follows: (Actual count of untreated host / Actual count of each challenge) and then expected synergy was calculated as follows: (Fold reduction of phage challenge* fold reduction of each antibiotic challenge).

\section{Adsorption assay}

A volume of $1 \mathrm{~mL}$ of either freshly grown purified survivor cultures, lysogen control or LB broth control were incubated with shaking for 30 min with $100 \mathrm{~mL}$ of diluted lambda-vir phage lysate of titer $10^{4} \mathrm{pfu} / \mathrm{mL}$. Subsequently, one hundred microliters of each tube after filtration by centrifugation was mixed with $300 \mathrm{~mL}$ of host overnight culture, inoculated in $5 \mathrm{~mL}$ of molten LB soft agar, and then overlay plates were prepared. Plaques were counted from plates after an overnight incubation at $37 € C$.Percent of adsorbed phages were then calculated as follows: ((Plaques count of blank- Plaques count of each sample) ${ }^{*} 100 /$ Phage plaques count of blank).

\section{Phage titer after overnight challenge}

The number of phage particles arising from phage challenge in the absence of antibiotic and in the presence of each antibiotic concentration tested for overnight quantification assay was determined using a phage plaque assay. Overnight cultures from the three challenges (phage alone and phage + antibiotic) were filtered using $0.45 \mu \mathrm{m}$ Basix ${ }^{\mathrm{TM}}$ Syringe Filters, PES, Sterile from Thermo Fisher Scientific to obtain phage lysates. Tenfold serial dilution was carried out in lysogeny broth (LB) in a final volume of $1 \mathrm{~mL}$. Lysates were titred using the standard double agar overlay technique. After overnight incubation, plaques were counted to calculate the number of phage particles in $\mathrm{pfu} / \mathrm{mL}$.

\section{Lysogeny detection}

The integration of HK97 into the host chromosome was confirmed via Polymerase Chain Reaction (PCR) two times for confirmation. Individual surviving colonies arising from PAS challenge with $1 / 4$ and $1 / 8 \mathrm{MIC}$, where we started seeing our first survivors, were purified by streaking. This was followed by colony PCR in which primers were designed to amplify the phage-host junction. Each $25 \mathrm{~mL}$ PCR reaction contained $1 \mathrm{ml}$ of each primer, $2.5 \mathrm{~mL}$ 10x DNA polymerase buffer, $0.5 \mathrm{~mL}$ dNTPs, $0.25 \mathrm{~mL}$ Taq DNA polymerase, $1 \mu \mathrm{L}$ of purified survivor grown overnight and the remaining volume was completed with nuclease free water. All PCR reagents were obtained from FroggaBio (NY, USA). Primer sequences are available in Key resources table (Al-Anany et al., 2021).

\section{Quantitative PCR}


qPCR was carried out as previously described (Al-Anany et al., 2021). Briefly. Freshly grown culture was challenged with phage at a $\mathrm{MOI}$ of at least 10 in the absence and presence of gentamicin antibiotic at $1 / 2$ MIC in three biological replicates each has 5 replicates. Challenges were incubated with shaking at $37 € C$ and $130 \mathrm{rpm}$ (Ecotron, Infors $\mathrm{HT}$, Quebec, Canada). One replicate of each of the two challenges was removed after 1 , $2,3,4$, and $18 \mathrm{~h}$ of exposure. To remove free floating DNA from lysed cells, challenges were treated with DNase, followed by addition of EDTA and heat inactivation at $75 € C$ for 10 min. Genomic extraction was carried out using the Monarch Genomic DNA Purification Kit (New England Biolab, MA, USA). The E. coli housekeeping gene cys $G$ was used as a control for quality of the DNA extraction in that sample. Each sample was amplified using primers designed to detect cysG, Hk97 lysogen integration site, and nonlysogens. PowerUp SYBR Green Master Mix (Applied Biosystems, MA, USA), BioRad CFX96 Touch Real Time Detection System, and CFX Manager 3.1.1517.0823. (California, USA) were used to carry out qPCR. QPCR cycling mode as follows; initial denaturation at $94^{\circ} \mathrm{C}$ for $2 \mathrm{~min}$. This was then followed by 40 cycles as follow: denaturation at $95 € \mathrm{C}$ for $15 \mathrm{~s}$ and annealing/extension at $60 € C$ for $1 \mathrm{~min}$. Melt curve generated by heating from $65 € \mathrm{C}$ to $95 € C$ in $0.5 € C$ increments per second. Primer sequences are available in Key resources table (Al-Anany et al., 2021).

\section{Quantification and statistical analysis}

All the statistical details of experiments can be found in the figure legends, figures and results. Quantitative values were expressed by mean $\pm S D$. They were compared by $t$ test, one-way ANOVA, two-way ANOVA, Tukey post hoc when appropriate with $P$ value $\leq 0.05$ is considered significant. All statistical analysis was done using GraphPad Prism 9.2.0 (GraphPad Software, Inc., CA, US). 
Al-Anany, A.M., Fatima, R., and Hynes, A.P. (2021). Temperate phage-antibiotic synergy eradicates bacteria through depletion of lysogens. Cell Rep. 35, 109172.

Amyes, S.G.B., and Smith, J.T. (1974). Trimethoprim action and its analogy with thymine starvation. Antimicrob. Agents Chemother. 5, 169-178.

Baharoglu, Z., and Mazel, D. (2011). Vibrio cholerae triggers SOS and mutagenesis in response to a wide range of antibiotics: a route towards multiresistance. Antimicrob. Agents Chemother. 55, 2438-2441.

Bedi, M.S., Verma, V., and Chhibber, S. (2009). Amoxicillin and specific bacteriophage can be used together for eradication of biofilm of Klebsiella pneumoniae B5055. World J. Microbiol. Biotechnol. 25, 1145-1151.

Bellio, P., Brisdelli, F., Perilli, M., Sabatini, A., Bottoni, C., Segatore, B., Setacci, D., Amicosante, G., and Celenza, G. (2014). Curcumin inhibits the SOS response induced by levofloxacin in Escherichia coli . Phytomedicine 21, 430-434.

Boling, L., Cuevas, D.A., Grasis, J.A., Kang, H.S., Knowles, B., Levi, K., Maughan, H., McNair, K., Rojas, M.I., Sanchez, S.E., et al. (2020). Dietary prophage inducers and antimicrobials: toward landscaping the human gut microbiome. Gut Microbes 1-14.

Bycroft BW, R.S. (1985). The Molecular basis for the mode of action of beta-lactam antibiotics and mechanisms of resistance. Pharm. Res. 2, p.3-14.

Chan, B.K., Sistrom, M., Wertz, J.E., Kortright, K.E., Narayan, D., and Turner, P.E. (2016). Phage selection restores antibiotic sensitivity in MDR Pseudomonas aeruginosa . Sci. Rep. 6, 1-8.

Chaudhry, W.N., Concepcion-Acevedo, J., Park, T., Andleeb, S., Bull, J.J., and Levin, B.R. (2017). Synergy and order effects of antibiotics and phages in killing Pseudomonas aeruginosa biofilms. PLoS One 12, 1-16.

Chhibber, S., Kaur, T., and Kaur, S. (2013). Co-therapy using lytic bacteriophage and Linezolid: effective treatment in eliminating methicillin resistant Staphylococcus aureus (MRSA) from diabetic foot infections. PLoS One 8, 1-11.

Comeau, A.M., Tétart, F., Trojet, S.N., Prère, M.-F., and Krisch, H.M. (2007). Phageantibiotic synergy (PAS): $\beta$-Lactam and quinolone antibiotics stimulate virulent phage growth. PLoS One 2, e799.

Cooper, C.J., Khan Mirzaei, M., and Nilsson, A.S. (2016). Adapting drug approval pathways for bacteriophage-based therapeutics. Front. Microbiol. 7, 1209.

Coulter, L.B., McLean, R.J.C., Rohde, R.E., and Aron, G.M. (2014). Effect of bacteriophage infection in combination with tobramycin on the emergence of resistance in Escherichia coli and Pseudomonas aeruginosa biofilms. Viruses 6, 3778-3786.

Czyz, A., Los, M., Wrobel, B., and Wegrzyn, G. (2001). Inhibition of spontaneous induction of lambdoid prophages in Escherichia coli cultures: simple procedures with 
possible biotechnological applications. BMC Biotechnol. 1, 1-5.

Danovaro, R., and Corinaldesi, C. (2003). Sunscreen products increase virus production through prophage induction in marine bacterioplankton. Microb. Ecol. 2003452 45, 109-118.

Davis, C.M., Mccutcheon, J.G., and Dennis, J.J. (2021). Aztreonam lysine increases the activity of phages E79 and phiKZ against Pseudomonas aeruginosa PA01 . 9, 1-19.

Dickey, J., Ronique, V., and Id, P. (2019). Adjunct phage treatment enhances the effectiveness of low antibiotic concentration against Staphylococcus aureus biofilms in vitro. PLoS One 14, 1-17.

Drimmelen, G.C. Van (1959). Bacteriology bacteriophage typing applied to strains of brucella organisms. Nature 184, 1079.

Drulis-Kawa, Z., Majkowska-Skrobek, G., Maciejewska, B., Delattre, A.-S., and Lavigne, R. (2013). Learning from bacteriophages - advantages and limitations of phage and phage-encoded protein applications. Curr. Protein Pept. Sci. 13, 699-722.

Duplessis, C., Warawa, J.M., Lawrenz, M.B., Henry, M., and Biswas, B. (2021). Successful intratracheal treatment of phage and antibiotic combination therapy of a multi-drug resistant Pseudomonas aeruginosa murine model. Antibiotics 10, 1-16.

Engeman, E., Freyberger, H.R., Corey, B.W., Ward, A.M., He, Y., Nikolich, M.P., Filippov, A.A., Tyner, S.D., and Jacobs, A.C. (2021). Synergistic killing and resensitization of Pseudomonas aeruginosa to antibiotics by phage-antibiotic combination treatment. Pharmaceuticals 14, 1-15.

Fortier, L.C., and Sekulovic, O. (2013). Importance of prophages to evolution and virulence of bacterial pathogens. Virulence 4, 354-365.

Ghosh, D., Roy, K., Williamson, K.E., Srinivasiah, S., Wommack, K.E., and Radosevich, M. (2009). Acyl-homoserine lactones can induce virus production in lysogenic bacteria: An alternative paradigm for prophage induction. Appl. Environ. Microbiol. 75, 71427152.

Goerke, C., Koller, J., and Wolz, C. (2006). Ciprofloxacin and trimethoprim cause phage induction and virulence modulation in Staphylococcus aureus. Antimicrob. Agents Chemother. 50, 171-177.

Gordillo Altamirano, F.L., and Barr, J.J. (2019). Phage therapy in the postantibiotic era. Clin. Microbiol. Rev. 32.

Hagens, S., Habel, A., and Bläsi, U. (2006). Augmentation of the antimicrobial efficacy of antibiotics by filamentous phage. Microb. Drug Resist. 12, 164-168.

Hendrix, R.W. (1983). Lambda II Cold Spring Harbor monograph series (Cold Spring Harbor Laboratory).

Hendrix, R.W., Bamford, D., Casjens, S., Christie, G., Duda, B., Grimes, S.N., Hatfull, G., Jardine, P., Kuhn, A., Miller, E., et al. (2013). Bacteriophages. Uniw. Śląski 343- 
354.

Herskowitz, I., and Hagen, D. (1980). The Lysis-Lysogeny decision of phage $\lambda$ : explicit programming and responsiveness. Annu. Rev. Genet. 14, 399-445.

Howard-Varona, C., Hargreaves, K.R., Abedon, S.T., and Sullivan, M.B. (2017). Lysogeny in nature: mechanisms, impact and ecology of temperate phages. ISME J. 11, 1511-1520.

Imamovic, L., and Muniesa, M. Characterizing RecA-Independent Induction of Shiga toxin2-Encoding Phages by EDTA Treatment. PLoS One 7, 1-15.

Iqbal, M., Narulita, E., Zahra, F., and Murdiyah, S. (2020). Effect of phage-antibiotic synergism (PAS) in increasing antibiotic inhibition of bacteria caused of foodborne diseases. J. Infect. Dev. Ctries. 14, 488-493.

Jansen, M., Wahida, A., Latz, S., Krüttgen, A., Häfner, H., Buhl, E.M., Ritter, K., and Horz, H.P. (2018). Enhanced antibacterial effect of the novel T4-like bacteriophage KARL-1 in combination with antibiotics against multi-drug resistant Acinetobacter baumannii. Sci. Rep. 8, 1-12.

Kaur, S., and Chhibber, S. (2021). A mouse air pouch model for evaluating the antibacterial efficacy of phage MR-5 in resolving skin and soft tissue infection induced by methicillin-resistant Staphylococcus aureus . Folia Microbiol. (Praha). 66, 959-972.

Kebriaei, R., Lev, K., Morrisette, T., Stamper, K.C., Abdul-Mutakabbir, J.C., Lehman, S.M., Morales, S., and Rybak, M.J. (2020). Bacteriophage-antibiotic combination strategy: an alternative against methicillin-resistant phenotypes of Staphylococcus aureus. Antimicrob. Agents Chemother. 64, 1-6.

Kim, J., Hur, J.I., Ryu, S., and Jeon, B. (2021). Bacteriophage-mediated modulation of bacterial competition during selective enrichment of Campylobacter . Microbiol. Spectr. 9, 1-9.

Kim, M., Jo, Y., Hwang, Y.J., Hong, H.W., Hong, S.S., Park, K., and Myung, H. (2018). Phage antibiotic synergy via delayed lysis. Appl. Environ. Microbiol. 84, e02085-18.

Kirby, A.E. (2012). Synergistic action of gentamicin and bacteriophage in a continuous culture population of Staphylococcus aureus. PLoS One 7, 1-9.

Knezevic, P., Curcin, S., Aleksic, V., Petrusic, M., and Vlaski, L. (2013). Phageantibiotic synergism: a possible approach to combatting Pseudomonas aeruginosa. Res. Microbiol. 164, 55-60.

Kohanski MA et al. (2007). A common mechanism of cellular death induced by bactericidal antibiotics. Cell 130, 797-810.

Kropinski, A.M., Mazzocco, A., Waddell, T.E., Lingohr, E., and Johnson, R.P. (2009). Enumeration of bacteriophages by double agar overlay plaque assay (Humana Press).

Labrie, S.J., Samson, J.E., and Moineau, S. (2010). Bacteriophage resistance mechanisms. Nat. Rev. Microbiol. 8, 317-327. 
Lemire, S., Figueroa-Bossi, N., and Bossi, L. (2011). Bacteriophage crosstalk: coordination of prophage induction by trans-acting antirepressors. PLoS Genet. 7, e1002149.

Lewin, C.S., and Amyes, S.G.B. (1991). The role of the SOS response in bacteria exposed to zidovudine or trimethoprim. J. Med. Microbiol 34, 329-332.

Li, X., Hu, T., Wei, J., He, Y., Abdalla, A.E., Wang, G., Li, Y., and Teng, T. (2021). Characterization of a novel bacteriophage Henu2 and evaluation of the synergistic antibacterial activity of phage-antibiotics. Antibiotics 10,1-14.

Liu, C.G., Green, S.I., Min, L., Clark, J.R., Salazar, K.C., Terwilliger, A.L., Kaplan, H.B., Trautner, B.W., Ramig, R.F., and Maresso, A.W. (2020). Phage-antibiotic synergy is driven by a unique combination of antibacterial mechanism of action and stoichiometry. BioRxiv 11, 1-19.

Liu, S., Zhao, Y., Hayes, A., Hon, K., Zhang, G., Bennett, C., Hu, H., Finnie, J., Morales, S., Shearwin, L., et al. (2021). Overcoming bacteriophage insensitivity in Staphylococcus aureus using clindamycin and azithromycinat subinhibitory concentrations. Allergy 76, 3446-3458.

López, E., Domenech, A., Ferrándiz, M.-J., Frias, M.J., Ardanuy, C., Ramirez, M., García, E., Liñares, J., and de la Campa, A.G. (2014). Induction of prophages by fluoroquinolones in Streptococcus pneumoniae: implications for emergence of resistance in genetically-related clones. PLoS One 9, e94358.

Luthe, T., Hünnefeld, M., Gätgens, C., Milke, L., Wiechert, J., Wittmann, J., Moraru, C., Marienhagen, J., and Frunzke, J. (2021). Aminoglycoside antibiotics inhibit phage infection by blocking an early step of the phage infection cycle. BioRxiv 1-31.

Maiques, E., Úbeda, C., Campoy, S., Salvador, N., Lasa, Í., Novick, R.P., Barbé, J., and Penadés, J.R. (2006). Beta-lactam antibiotics induce the SOS response and horizontal transfer of virulence factors in Staphylococcus aureus. J. Bacteriol. 188, 2726-2729.

Malik, S., Nehra, K., and Rana, J.S. (2021). Bacteriophage cocktail and phage antibiotic synergism as promising alternatives to conventional antibiotics for the control of multidrug-resistant uropathogenic Escherichia coli . Virus Res. 302, 198496.

Manohar, P., Royam, M.M., Loh, B., Bozdogan, B., Nachimuthu, R., and Leptihn, S. (2022). Synergistic effects of phage-antibiotic combinations against Citrobacter amalonaticus . ACS Publ. 8, 59-65.

Martinez-Irujo, J.J., Villahermosa, M.L., Alberdi, E., and Santiago, E. (1996). A checkerboard method to evaluate interactions between drugs. Biochem. Pharmacol. 51, 635-644.

Miller, C., Thomsen, L.E., Gaggero, C., Mosseri, R., Ingmer, H., and Cohen, S.N. (2004). SOS response induction by $\beta$-lactams and bacterial defense against antibiotic lethality. Science. 305, 1629-1631.

Monteiro, R., Pires, D.P., Costa, A.R., and Azeredo, J. (2018). Phage therapy: going temperate? Trends Microbiol. 27, 368-378. 
Moradpour, Z., Yousefi, N., Sadeghi, D., and Ghasemian, A. (2020). Synergistic bactericidal activity of a naturally isolated phage and ampicillin against urinary tract infecting Escherichia coli O157. Iran. J. Basic Med. Sci. 23, 257.

N H Georgopapadakou, B A Dix, P Angehrn, A Wick, and G.L.O. (1987). Monocyclic and tricyclic analogs of quinolones: mechanism of action.

Van Nieuwenhuyse, B., Galant, C., Brichard, B., Docquier, P.-L., Djebara, S., Pirnay, J.P., Van Der Linden, D., Merabishvili, M., and Chatzis, O. (2021). A case of in situ phage therapy against Staphylococcus aureus in a bone allograft polymicrobial biofilm infection: outcomes and phage-antibiotic interactions. Viruses 13, 1-12.

Oechslin, F., Piccardi, P., Mancini, S., Gabard, J., Moreillon, P., Entenza, J.M., Resch, G., and Que, Y.A. (2017). Synergistic interaction between phage therapy and antibiotics clears Pseudomonas aeruginosa infection in endocarditis and reduces virulence. J. Infect. Dis. 215, 703-712.

Oppenheim, A.B., Kobiler, O., Stavans, J., Court, D.L., and Adhya, S. (2005). Switches in bacteriophage Lambda development. Annu. Rev. Genet. 39, 409-429.

Pacios, O., Fernández-García, L., Bleriot, I., Blasco, L., González-Bardanca, M., López, M., Fernández-Cuenca, F., Oteo, J., Pascual, Á., Martínez-Martínez, L., et al. (2021). Enhanced antibacterial activity of repurposed mitomycin $\mathrm{C}$ and imipenem in combination with the lytic phage vB_KpnM-VAC13 against clinical isolates of Klebsiella pneumoniae. Antimicrob. Agents Chemother. 65.

Rozanov, D. V, Ari, R.D.', and Sineoky, S.P. (1998). RecA-Independent Pathways of Lambdoid Prophage Induction in Escherichia coli . J. Bacteriol. 180, 6306-6315.

Ryan, E.M., Alkawareek, M.Y., Donnelly, R.F., and Gilmore, B.F. (2012). Synergistic phage-antibiotic combinations for the control of Escherichia coli biofilms in vitro. FEMS Immunol. Med. Microbiol. 65, 395-398.

S. Shiba, A. Terawaki, T.T.\& J.K. (1952). Selective inhibition of formation of deoxyribonucleic acid in Escherichia coli by mitomycin C. Nature 183, 1056-1057.

Save, J., Que, Y.-A., Entenza, J.M., Kolenda, C., Laurent, F., and Resch, G. (2022). Bacteriophages combined with subtherapeutic doses of flucloxacillin act synergistically against Staphylococcus aureus experimental infective endocarditis. J. Am. Heart Assoc. 11, 23080.

Shkilnyj, P., and Koudelka, G.B. (2007). Effect of salt shock on stability of imm434 lysogens. J. Bacteriol. 189, 3115-3123.

Simmons, L.A., Foti, J.J., Cohen, S.E., and Walker, G.C. (2008). The SOS regulatory network. EcoSal Plus 3, 1-48.

Simon, K., Pier, W., Krüttgen, A., and Horz, H.-P. (2021). Synergy between phage Sb-1 and oxacillin against methicillin-resistant Staphylococcus aureus . Antibiotics 10,1-9.

Southward, C.M., and Surette, M.G. (2002). The dynamic microbe: green fluorescent protein brings bacteria to light. Mol. Microbiol. 45, 1191-1196. 
Styles, K.M., Thummeepak, R., Leungtongkam, U., Smith, S.E., Christie, G.S., Millard, A., Moat, J., Dowson, C.G., Wellington, E.M.H., Sitthisak, S., et al. (2020). Investigating bacteriophages targeting the opportunistic pathogen Acinetobacter baumannii . Antibiotics 9, 1-19.

Sun, X., Göhler, A., Heller, K.J., and Neve, H. (2006). The Itp gene of temperate Streptococcus thermophilus phage TP-J34 confers superinfection exclusion to Streptococcus thermophilus and Lactococcus Lactis . Virology 350, 146-157.

Sutcliffe, S.G., Shamash, M., Hynes, A.P., and Maurice, C.F. (2021). Common Oral Medications Lead to Prophage Induction in Bacterial Isolates from the Human Gut. Viruses 13, 455.

Torres-Barceló, C., Arias-Sánchez, F.I., Vasse, M., Ramsayer, J., Kaltz, O., and Hochberg, M.E. (2014). A window of opportunity to control the bacterial pathogen Pseudomonas aeruginosa combining antibiotics and phages. PLoS One 9.

Torres-Barceló, C., Gurney, J., Gougat-Barberá, C., Vasse, M., and Hochberg, M.E. (2018). Transient negative effects of antibiotics on phages do not jeopardise the advantages of combination therapies. FEMS Microbiol. Ecol. 94.

Verma, V., Harjai, K., and Chhibber, S. (2009). Characterization of a T7-Like lytic bacteriophage of Klebsiella pneumoniae b5055: A potential therapeutic agent. Curr. Microbiol. 59, 274-281.

Vondrejs, D.V.\& V. (1974). Induction of bacteriophage lambda with oxolinic and nalidixic Acid. Folia Microbiol. (Praha). 19, 390-393.

Wang, L., Tkhilaishvili, T., Trampuz, A., and Gonzalez Moreno, M. (2020). Evaluation of Staphylococcal Bacteriophage Sb-1 as an Adjunctive Agent to Antibiotics Against Rifampin-Resistant Staphylococcus aureus Biofilms. Front. Microbiol. 11, 2700.

Wiegand, I., Hilpert, K., and Hancock, R.E.W. (2008). Agar and broth dilution methods to determine the minimal inhibitory concentration (MIC) of antimicrobial substances. Nat. Protoc. 3, 163-175.

Yasukawa, K., Kitanaka, S., and Seo, S. (2002). Inhibitory effect of stevioside on tumor promotion by 12-O-tetradecanoylphorbol-13-acetate in two-stage carcinogenesis in mouse skin. Biol. Pharm. Bull. 25, 1488-1490.

Yilmaz, C., Colak, M., Yilmaz, B.C., Ersoz, G., Kutateladze, M., and Gozlugol, M. (2013). Bacteriophage therapy in implant-related infections: An experimental study. J. Bone Jt. Surg. - Ser. A 95, 117-125.

Zaslaver, A., Bren, A., Ronen, M., Itzkovitz, S., Kikoin, I., Shavit, S., Liebermeister, W., Surette, M.G., and Alon, U. (2006). A comprehensive library of fluorescent transcriptional reporters for Escherichia coli. Nat. Methods 200638 3, 623-628. 


\section{Supplementary Files}

This is a list of supplementary files associated with this preprint. Click to download.

- 2Supplementary.pdf 